F. Halter-Koch

Nagoya Math. J.

Vol. 123 (1991), 141-151

\title{
ON A CLASS OF INSOLUBLE BINARY QUADRATIC DIOPHANTINE EQUATIONS
}

\author{
FRANZ HALTER-KOCH
}

\section{§. Introduction}

The binary quadratic diophantine equation

$$
\left|x^{2}-n y^{2}\right|=t
$$

is of interest in the class number problem for real quadratic number fields and was studied in recent years by several authors (see [4], [5], [2] and the literature cited there).

To be precise, for a positive square-free integer $n$, we set

$$
\sigma_{n}= \begin{cases}1, & \text { if } n \not \equiv 1 \bmod 4 ， \\ 2, & \text { if } n \equiv 1 \bmod 4 ;\end{cases}
$$

a solution $(x, y) \in \mathbf{Z}$ of the diophantine equation

$$
\left|x^{2}-n y^{2}\right|=\sigma_{n}^{2} t
$$

is called primitive, if $(x, y) \mid \sigma_{n}$, where $(x, y)$ denotes the g.c.d. of $x$ and $y$. The reason for this terminology will become clear from the theory of quadratic orders, to be explained in $\S 1$.

R. A. Mollin [4] proved, generalizing previous results by Yokoi [5] and others, the following criterion.

Proposition 0. Let $s, t, r$ be integers such that $n=(s t)^{2}+r>5$ is squarefree and the following conditions are satisfied:

(1) $s \geq 1, t \geq 2$ and $(t, r)=1$;

(2) $r \mid 4 s$, and $-s t<r \leq s t$;

(3) If $n \equiv 1 \bmod 4$, then $|r| \in\{1,4\}$.

(4) If $|r|=4$, then $s \geq 2$.

(5) If $r=1$, then $\mathrm{s} \geq 3$ and $2 \mid$ st.

Then the diophantine equation $\left|x^{2}-n y^{2}\right|=\sigma_{n}^{2} t$ has a primitive solution if

Received November 13, 1990. 
and only if $n=7, t=3$.

Actually, the result as given in [4], is formally stronger than Proposition 0 ; there it is asserted, that the diophantine equation has no nontrivial solutions (in a sense precised there). To obtain Mollin's result, we must apply Proposition 0 for all $t^{\prime}>1$ such that $t=t^{\prime} u^{2}$ for some $u \in \mathbf{N}$.

In [2], we derived a general method to handle such equations using continued fractions, and we claimed [2, p. 92] that an application of these techniques would lead to a simple proof and a generalization of Proposition 0. J. B. Leicht (Heidelberg) pointed out to me that this is not quite correct: The techniques of [2] do only work if $\sigma_{n} t<\sqrt{n}$, and there are two cases of Proposition 0 in which this condition is violated:

$$
\begin{array}{ll}
s=1, & r=-1, \quad n=t^{2}-1 ; \\
s=1, & r=-2, \quad n=t^{2}-2 .
\end{array}
$$

In this paper, we develop different techniques which, among others, also cover these cases. We consider the diophantine equation as a norm equation, and then the ideal theory of quadratic orders becomes available for the problem $(\S 1)$. In $\S 2$ we prove a criterion for certain ideals to be reduced (Theorem 1) and a general reduction statement (Theorem 2). In $\S 3$ we reformulate these Theorems for diophantine equations. Finally, in $\S 4$, we give some applications for discriminants of Richaud-Degerttype; thereby we restrict ourselves to those cases, which cannot be settled with the methods of [2].

\section{$\S 1$. Preliminaries on quadratic orders}

In this section we recall some well-known facts about quadratic orders and formulate them in a manner which will be useful later on; for proofs see [1] or [3] (but note that the notions of [3] are slightly different from ours).

A positive integer $D$ is called a discriminant, if $D$ is not a square and $D \equiv 0$ or $1 \bmod 4$; in this paper, $D$ always denotes a discriminant. We set

$$
\omega_{D}= \begin{cases}\frac{1}{2} \sqrt{D}, & \text { if } D \equiv 0 \bmod 4, \\ \frac{1}{2}(1+\sqrt{D}), & \text { if } D \equiv 1 \bmod 4,\end{cases}
$$


and

$$
\mathscr{R}_{D}=\mathbf{Z} \oplus \mathbf{Z} \omega_{D}
$$

$\mathscr{R}_{D}$ is an order in the quadratic number field $\mathbf{Q}(\sqrt{D})$. If $D_{0}$ is the discriminant of $\mathbf{Q}(\sqrt{D})$, then

$$
D=D_{0} f_{D}^{2}
$$

for some $f_{D} \in \mathbf{N} ; f_{D}$ is called the conductor associated with $D$.

Every $\xi \in \mathscr{R}_{D}$ has a unique representation in the form

$$
\xi=\frac{b+e \sqrt{D}}{2}
$$

where $b, e \in \mathbf{Z}$ and $b \equiv e D \bmod 2$; we call

$$
\mathscr{N}(\xi)=\frac{b^{2}-e^{2} D}{4} \in \mathbf{Z}
$$

the norm of $\xi$. An element $\xi \in \mathscr{R}_{D}$ is called primitive, if $m^{-1} \xi \notin \mathscr{R}_{D}$ for all integers $m \geq 2$. Obviously, $\xi \in \mathscr{R}_{D}$ is primitive if and only if either

$$
D \equiv 0 \bmod 4, \quad \xi=x+y \sqrt{\frac{D}{4}}, \quad x, y \in \mathbf{Z}, \quad(x, y)=1
$$

or

$$
D \equiv 1 \bmod 4, \quad \xi=\frac{x+y \sqrt{D}}{2}, \quad x, y \in \mathbf{Z}, \quad x \equiv y \bmod 2,(x, y) \mid 2 .
$$

For an ideal $(0) \neq J \triangleleft \mathscr{R}_{D}$ we call

$$
\mathscr{N}(J)=\left(\mathscr{R}_{D}: J\right) \in \mathbf{N}
$$

the norm of $J$; $J$ is called primitive, if $m^{-1} J \not \subset \mathscr{R}_{D}$ for all integers $m \geq 2$. If $J=\xi \mathscr{R}_{D}$ is a principal ideal, then $\mathscr{N}(J)=|\mathscr{N}(\xi)|$, and $J$ is primitive if and only if $\xi$ is primitive. Let $\Omega(D)$ be the set of all norms of primitive principal ideals of $\mathscr{R}_{D}$. Using this terminology, we rephrase the question about the solubility of the diophantine equations under consideration as follows.

Proposition 1. If $D$ is a discriminant and $t$ is a positive integer, then the following two assertions are equivalent:

a) $t \in \Omega(D)$

b) The diophantine equation 


$$
\begin{cases}\left|x^{2}-\frac{D}{4} y^{2}\right|=t, & \text { if } D \equiv 0 \bmod 4, \\ \left|x^{2}-D y^{2}\right|=4 t, & \text { if } D \equiv 1 \bmod 4\end{cases}
$$

has a solution $(x, y) \in \mathbf{Z}^{2}$ satisfying

$$
\begin{cases}(x, y)=1, & \text { if } D \equiv 0 \bmod 4, \\ (x, y) \mid 2, & \text { if } D \equiv 1 \bmod 4 .\end{cases}
$$

An ideal $(0) \neq J \triangleleft \mathscr{R}_{D}$ is called regular, if $\mathscr{R}_{D}=\left\{x \in \mathbf{Q}(\sqrt{\bar{D}}) \mid x_{J} \subset J\right\}$. Any regular ideal is invertible. Any principal ideal and any ideal $J$ of $\mathscr{R}_{D}$ such that $\left(\mathscr{N}(J), f_{D}\right)=1$ is regular. In this paper we shall mainly be concerned with ideals $J$ such that $\left(\mathscr{N}(J), f_{D}\right)=1$.

The primitive ideals of $\mathscr{R}_{D}$ are precisely the Z-modules of the form

$$
J=\mathbf{Z} a \oplus \mathbf{Z} \frac{b+\sqrt{D}}{2}
$$

where $a, b \in \mathbf{Z}, a>0$ and $4 a \mid b^{2}-D$. In this representation, $a=\mathscr{N}(J)$ is uniquely determined by $J$, while $b$ is only determined modulo $2 a$. If $J$ is as above, then $J$ is regular if and only if $\left(a, b,\left(b^{2}-D\right) / 4 a\right)=1$.

For lack of a suitable reference, we give a proof of the following simple result concerning ideals whose norm divides the discriminant.

Lemma 1. Let $D$ be a discriminant and $r$ a positive integer such that $r \mid D$ and $4 \nmid r$. Then there exists exactly one primitive ideal $J \triangleleft \mathscr{R}_{D}$ such that $\mathscr{N}(J)=r$.

Proof. Since $4 \nmid r$, we have either $4 r \mid D$ or $4 r \mid r^{2}-D$, and we set

$$
J= \begin{cases}\mathrm{Z} r \oplus \mathbf{Z} \frac{\sqrt{D}}{2}, & \text { if } 4 r \mid D, \\ \mathbf{Z} r \oplus \mathbf{Z} \frac{r+\sqrt{D}}{2}, & \text { if } 4 r \nmid D .\end{cases}
$$

Then $J$ is a primitive ideal of $\mathscr{R}_{D}$, and $\mathscr{N}(J)=r$.

If $I=\mathbf{Z r} \oplus \mathbf{Z}(b+\sqrt{D}) / 2$ is a primitive ideal of $\mathscr{R}_{D}$, where $0 \leq b$ $<2 r, 4 r \mid b^{2}-D$, then $r \mid D$ implies $r \mid b$ and therefore $b=0$ or $b=r$. If there were two primitive ideals in $\mathscr{R}_{D}$ with norm $r$, then $I_{1}=\mathbf{Z r} \oplus$ $\mathrm{Z}(\sqrt{D} / 2)$ and $I_{2}=\mathrm{Z} r \oplus \mathrm{Z}(r+\sqrt{D}) / 2$ both were ideals, whence $4 r \mid D$ and $4 r \mid r^{2}-D$; this implies $4 r \mid r^{2}$ and hence $4 \mid r$, contradicting the assumption that $r$ is square-free. 
An ideal $(0) \neq J \triangleleft \mathscr{R}_{D}$ is called reduced, if it is primitive, regular, and has a representation of the form

$$
J=\mathbf{Z} a \oplus \mathbf{Z} \frac{b+\sqrt{D}}{2}
$$

such that

$$
0<\sqrt{D}-b<2 a<\sqrt{\bar{D}}+b ;
$$

note that these conditions also determine $b$ uniquely. If $J$ is a reduced ideal of $\mathscr{R}_{D}$, then $\mathscr{N}(J)<\sqrt{D}$. If $J$ is a primitive regular ideal of $\mathscr{R}_{D}$ and $\mathscr{N}(J)<\frac{1}{2} \sqrt{D}$, then $J$ is reduced.

Two ideals $J_{1}, J_{2} \triangleleft \mathscr{R}_{D}$ are called equivalent, if there exist elements $\beta_{1}, \beta_{2} \in \mathscr{R}_{D} \backslash\{0\}$ such that $\beta_{1} J_{1}=\beta_{2} J_{2}$.

If $J=\mathbf{Z} a \oplus \mathbf{Z}(b+\sqrt{D}) / 2$ is a primitive ideal of $\mathscr{R}_{D}(a, b \in \mathbf{Z}, a>0$, $\left.4 a \mid b^{2}-D\right)$, then its Lagrange neighbour $J^{+}$is defined by

$$
J^{+}=\mathbf{Z} a^{+} \oplus \mathbf{Z} \frac{b^{+}+\sqrt{D}}{2}
$$

where

$$
b^{+}=-b+2 a\left[\frac{b+\sqrt{D}}{2 a}\right] \text { and } a^{+}=\frac{D-b^{+2}}{4 a} .
$$

$J^{+}$is an ideal of $\mathscr{R}_{D}$, equivalent to $J$, and if $J$ is regular (reduced), then $J^{+}$is also regular (reduced). Let $\left(J_{n}\right)_{n \geq 0}$ be defined by $J_{0}=J$ and $J_{n+1}=$ $J_{n}^{+}$. The sequence $\left(J_{n}\right)_{n \geq 0}$ becomes ultimately periodic, and if $J$ is regular, it contains all reduced ideals equivalent to $J$. The sequence $\left(J_{n}\right)_{n \geq 0}$ can be calculated by means of the continued fraction algorithm as follows: If

$$
\xi=\frac{b+\sqrt{D}}{2 a}=\left[b_{0}, b_{1}, b_{2}, \cdots\right]
$$

is the simple continued fraction expansion of $\xi$ and, for $\nu \geq 0$,

$$
\xi_{\nu}=\left[b_{\nu}, b_{\nu+1}, \cdots\right]=\frac{P_{\nu}+\sqrt{D}}{2 Q_{\nu}},
$$

where $P_{\nu} \in \mathbf{Z}$ and $Q_{\nu} \in \mathbf{N}$, then

$$
J_{\nu}=\mathbf{Z} Q_{\nu} \oplus \mathbf{Z} \frac{P_{\nu}+\sqrt{D}}{2} .
$$

The case $J_{0}=\mathscr{R}_{D}$ is of particular interest: If 


$$
\omega_{D}=\left[b_{0}, \overline{b_{1}, \cdots, b_{l}}\right],
$$

$l$ is the length of a primitive period and if, for $\nu \in\{1, \cdots, l\}$,

$$
\xi_{\nu}=\left[\overline{b_{\nu}, b_{\nu+1}, \cdots, b_{l}, b_{1}, \cdots, b_{\nu-1}}\right]=\frac{P_{\nu}+\sqrt{D}}{2 Q_{\nu}}
$$

then the set

$$
\Omega^{*}(D)=\left\{Q_{1}, \cdots, Q_{l}\right\}
$$

is precisely the set of norms of reduced principal ideals of $\mathscr{R}_{D}$.

\section{§2. Reduced ideals}

Theorem 1. Let $D=4 t^{2}+m$ be a discriminant, where $t$ and $m$ are integers such that $t>0,4 t \nmid m$ and either

$$
m \geq-4 t+2
$$

or

$$
m \geq-8 t+5, \quad m \neq 1 \bmod 4 t .
$$

Then any primitive regular ideal $J$ of $\mathscr{R}_{D}$ with $\mathscr{N}(J)=t$ is reduced.

Proof. If $m>0$, then $t<\frac{1}{2} \sqrt{D}$, and therefore any primitive regular ideal of $\mathscr{R}_{D}$ with norm $t$ is reduced.

Thus we may suppose that $m<0$. Let $J \triangleleft \mathscr{R}_{D}$ be a primitive regular ideal with $\mathscr{N}(J)=t$, and set

$$
J=\mathbf{Z} t \oplus \mathbf{Z} \frac{x+\sqrt{D}}{2},
$$

where $1 \leq x \leq 2 t$ and $x^{2} \equiv D \equiv m \bmod 4 t$. Since $4 t \nmid m$, we have $x<2 t$, and we must prove that

$$
0<\sqrt{D}-x<2 t<\sqrt{\bar{D}}+x
$$

i.e.,

$$
x^{2}<D<(2 t+x)^{2} \text { and }(2 t-x)^{2}<D .
$$

Since $m<0$, we always have $D<4 t^{2}<(2 t+x)^{2}$. If $m \geq-4 t+2$, then $x^{2} \leq(2 t-1)^{2},(2 t-x)^{2} \leq(2 t-1)^{2}$, and $(2 t-1)^{2}<4 t^{2}+m=D$.

If $m \not \equiv 1 \bmod 4 t$ and $m \geq-8 t+5$, then $2 \leq x \leq 2 t-2, x^{2} \leq(2 t-2)^{2}$, $(2 t-x)^{2} \leq(2 t-2)^{2}$ and $(2 t-2)^{2}<4 t^{2}+m=D$. 
TheOREM 2. Let $D=t^{2}+m$ be a discriminant, where $t>1$ and $m$ are integers such that either

$$
-2 t+1<m<2 t+1
$$

or

$$
-4 t+4<m<4 t+4, \quad m \neq \equiv 1 \bmod t .
$$

Let $J \triangleleft \mathscr{R}_{D}$ be a primitive regular ideal such that $\mathscr{N}(J)=t$, let $J^{+}$be the Lagrange neighbour of $J$, and $Q=\mathscr{N}\left(J^{+}\right)$.

Then $Q<\frac{1}{2} \sqrt{D}$, and $D-4 t Q \in \mathbf{Z}$ is a perfect square. In particular, $J^{+}$is reduced.

Proof. Suppose that $J=\mathbf{Z} t \oplus \mathbf{Z}(y+\sqrt{\bar{D}}) / 2$ where $y \in \mathbf{Z}, \quad y^{2} \equiv$ $D \bmod 4 t$ and $t<y \leq 3 t$; we consider first the case $m \neq 4 t$. Then we have $y \neq 3 t$ and therefore $t+1 \leq y \leq 3 t-1$. Moreover, if $m \neq \equiv 1 \bmod t$, then $y^{2} \not \equiv 1 \bmod t$, and therefore $t+2 \leq y \leq 3 t-2$. Since

$$
\begin{array}{ll}
t-1<\sqrt{\bar{D}}<t+1, & \text { if }-2 t+1<m<2 t+1, \\
t-2<\sqrt{\bar{D}}<t+2, & \text { if }-4 t+4<m<4 t+4,
\end{array}
$$

we obtain in any case

$$
1<\frac{y+\sqrt{D}}{2 t}<2
$$

and therefore

$$
J^{+}=\mathbf{Z} Q \oplus \mathbf{Z} \frac{P+\sqrt{D}}{2},
$$

where $P=2 t-y$ and $Q=\left(D-P^{2}\right) / 4 t$. We set $y^{2}=D+4 t z$, where $z \in \mathbf{Z}$, and obtain

$$
Q=y-t-z .
$$

If $m=4 t$, then $y=3 t$ and $J^{+}=\mathscr{R}_{D}$, so that in this case $Q=1, z=$ $2 t-1$ and again

$$
Q=y-t-z
$$

In any case we obtain

$$
y^{2}=D+4 t(y-t-Q)=4 t y+D-4 t^{2}-4 t Q,
$$

and therefore 


$$
y=2 t \pm \sqrt{D-4 t Q},
$$

whence $D-4 t Q$ must be a perfect square.

Suppose that $Q>\frac{1}{2} \sqrt{ } \bar{D}$; then we obtain

$$
z=y-t-Q<y-t-\frac{1}{2} \sqrt{D},
$$

and therefore

$$
y^{2}=D+4 t z<t^{2}+m+4 t y-4 t^{2}-2 t \sqrt{ } \bar{D},
$$

whence

$$
y^{2}-4 t y+\left(3 t^{2}+2 t \sqrt{\bar{D}}-m\right)<0 .
$$

This however can only occur when

$$
(2 t)^{2}-\left(3 t^{2}+2 t \sqrt{D}-m\right)=t^{2}-2 t \sqrt{D}+m>0,
$$

i.e., when $2 t \sqrt{D}<t^{2}+m$. Squaring this inequality gives

$$
4 t^{2} D=4 t^{4}+4 t^{2} m<t^{4}+2 t^{2} m+m^{2},
$$

and therefore

$$
0>3 t^{4}+2 t^{2} m-m^{2}=\left(3 t^{2}-m\right)\left(t^{2}+m\right),
$$

contradicting our assumptions on $m$ and $t$.

\section{§ 3. Diophantine equations}

In this section we reformulate Theorems 1 and 2 for diophantine equations. We do this using the set $\Omega(D)$; the final translation into the language of diophantine equations is given by Proposition 1 .

Theorem 1A. Let $D=4 t^{2}+m$ be a discriminant as in Theorem 1 , and suppose that $\left(t, f_{D}\right)=1$. Then we have $t \in \Omega(D)$ if and only if $t \in$ $\Omega^{*}(D)$.

Proof. Since $\left(t, f_{D}\right)=1$, any ideal $J$ of $\mathscr{R}_{D}$ satisfying $\mathscr{N}(J)=t$ is regular. Therefore the assertion follows from Theorem 1.

TheOREM 2A. Let $D=t^{2}+m$ be a discriminant as in Theorem 2, and suppose that $\left(t, f_{D}\right)=1$.

i) If $t \in \Omega(D)$, then there exists some $Q \in \Omega^{*}(D)$ such that $Q<\frac{1}{2} \sqrt{D}$, and the integer $D-4 t Q$ is a perfect square. 
ii) If $Q \in \Omega(D)$ is such that the integer $D-4 t Q$ is a perfect square and all primitive ideals $J \triangleleft \mathscr{R}_{D}$ with $\mathscr{N}(J)=Q$ are principal ideals, then $t \in \Omega(D)$.

iii) If $D-4 t$ is a perfect square, then $t \in \Omega(D)$.

Proof. i) Let $J \triangleleft \mathscr{R}_{D}$ be a primitive principal ideal such that $\mathscr{N}(J)$ $=t$; since $\left(t, f_{D}\right)=1, J$ is regular. By Theorem $2, Q=\mathscr{N}\left(J^{+}\right)<\frac{1}{2} \sqrt{D}$, and $D-4 t Q$ is a perfect square.

ii) If $D-4 t Q=P^{2}$ for some $P \in \mathrm{N}$, then the primitive ideals $J_{1}=$ $\mathbf{Z} Q \oplus \mathbf{Z}(P+\sqrt{D}) / 2$ and $J_{2}=\mathbf{Z} t \oplus \mathbf{Z}(-P+\sqrt{D}) / 2$ are equivalent by [3, Cor. 2]. By assumption, $J_{1}$ is principal, whence $J_{2}$ is principal, too, and therefore $t \in \Omega(D)$.

iii) follows from ii) with $Q=1$.

\section{§4. Discriminants of Richaud-Degert-type}

Proposition 2. Let $D=4 a^{2}+r$ be a discriminant, where $a$ and $r$ are integers such that $1 \leq|r|<a, r \mid a, r$ is square-free and $r \equiv 1 \bmod 4$.

i) If $r \neq 1$, then $a \notin \Omega(D)$.

ii) $2 a \in \Omega(D)$ if and only if either $4 a^{2}-8 a+r$ or $4 a^{2}-8 a|r|+r$ is a perfect square.

iii) $2 a \in \Omega\left(4 a^{2}+1\right)$ if and only if $a=2$.

Proof. Since $r$ is square-free, $\left(r, f_{D}\right)=1$. From [2] we obtain $\Omega^{*}(D)$ $=\{1, r, a \pm(r-1) / 4\}$ if $r>0$, and $\Omega^{*}(D)=\{1,|r|, a+(r-1) / 4\}$ if $r<0$.

i) follows from Theorem $1 \mathrm{~A}$ with $t=a, m=r$.

ii) We apply Theorem $2 \mathrm{~A}$ with $t=2 a, m=r$. If $2 a \in \Omega(D)$, then $D-4 t Q$ is a perfect square for one of the numbers $Q=1,|r|, a \pm(r-1) / 4$. If $Q=a \pm(r-1) / 4$, then $D-4 t Q<0$, and therefore it cannot be a perfect square. If $Q=|r|$, then $D-4 t Q=4 a^{2}-8 a|r|+r$, and if $Q=1$, then $D-4 t Q=4 a^{2}-8 a+r$.

For the converse suppose that, for $Q=1$ or $Q=|r|, D-4 t Q$ is a perfect square. By Lemma 1, there eixsts exactly one primitive ideal $J$ of $\mathscr{R}_{D}$ such that $\mathscr{N}(J)=Q$, and since $\{1,|r|\} \subset \Omega^{*}(D), J$ is principal. Now the assertion follow from Theorem $2 \mathrm{~A}$, ii).

iii) By ii), $2 a \in \Omega\left(4 a^{2}+1\right)$ if and only if $4 a^{2}-8 a+1=(2 a-2)^{2}-3$ is a perfect square, which is equivalent with $a=2$.

Proposition 3. Let $D=a^{2}+4 r$ be a discriminant, where $a$ and $r$ are integers such that $a \equiv 1 \bmod 2, a>1, r \mid a, r \neq-a$, and $r$ is square-free. 
i) $a \in \Omega(D)$ if and only if either $a^{2}-4 a+4 r$ or $a^{2}-4 a|r|+4 r$ is a perfect square.

ii) $a \in \Omega\left(a^{2}-4\right)$ if and only if $a=5$.

Proof. From $-a=\mathscr{N}\left(\frac{1}{2}\left(a+\sqrt{a^{2}+4 a}\right)\right)$ we obtain $a \in \Omega\left(a^{2}+4 a\right)$, and therefore we may suppose that $|r|<a$, and consequently $|r| \leq a / 3$. Since $r$ is square-free, $\left(r, f_{D}\right)=1$. From [2] we obtain $\Omega^{*}(D)=\{1, r\}$ if $r>0$, and $\Omega^{*}(D)=\{1,|r|, a+r-1\}$ if $r<0$.

We apply Theorem $2 \mathrm{~A}$ with $t=a, m=4 r$. If $a \in \Omega(D)$, then $D-$ $4 t Q$ is a perfect square for one of the numbers $Q=1,|r|, a+r-1$. If $Q=a+r-1$, then $D-4 t Q=-a(3 a+4 r)+4(a+r)<0$ cannot be a perfect square. If $Q=|r|$, then $D-4 t Q=a^{2}-4 a|r|+4 r$, and if $Q=1$, then $D-4 t Q=a^{2}-4 a+4 r$.

The converse is proved exactly as in Proposition 2.

ii) follows from i) with $r=-1$, observing that $a^{2}-4 a-4=$ $(a-1)^{2}-8$ is a perfect square if and only if $a=5$.

Proposition 4. Let $D=4\left(a^{2}+r\right)$ be a discriminant, where $a$ and $r$ are integers such that $a \geq 3, r \mid 2 a, r>-a$, and $r$ is square-free.

i) Suppose that either $2 \nmid a$ or $a^{2}+r$ is not a discriminant. Then $a \in \Omega(D)$ if and only if $a=r$.

ii) Suppose that $a^{2}+r$ is not a discriminant. Then $2 a \in \Omega(D)$ if and only if either $a^{2}-2 a+r$ or $a^{2}-2 a|r|+r$ is a perfect square. In particular:

If $r=1$, then $2 a \in \Omega(D)$;

if $r \in\{-1,2\}$, then $2 a \notin \Omega(D)$;

if $r=-2$, then $2 a \in \Omega(D)$ if and only if $a=3$.

Proof. From [2] we obtain $\Omega^{*}(D)=\{1, r\}$, if $r>0$, and $\Omega^{*}(D)=$ $\{1,2 a+r-1,|r|\}$, if $r<0$. Since $r$ is square-free, no odd prime divides $\left(a, f_{D}\right)$. Since $2 \mid f_{D}$ if and only if $a^{2}+r$ is a discriminant, we obtain $\left(a, f_{D}\right)=1$ in i) and $\left(2 a, f_{D}\right)=1$ in ii).

Now we proceed as in the proof of Proposition 2: We infer i) from Theorem $1 \mathrm{~A}$ with $t=a, m=4 r$, and ii) from Theorem $2 \mathrm{~A}$ with $t=2 a$, $m=4 r$.

\section{§5. An application}

We finish with an amusing application of the preceding theory, part of which was posed as a problem (cf. Bulletin dell' Association des 
Professeurs de Mathématiques no. 374, 1990, Problem no. 177).

Proposition 5. If $x$ and $y$ are positive integers such that, for some choice of the sign,

$$
c=\frac{x^{2}+y^{2}}{x y \pm 1}
$$

is an integer, then $c$ is either a perfect square, or $c=5$.

Proof. We suppose that $c=\left(x^{2}+y^{2}\right) /(x y \pm 1)$ is an integer and not a perfect square; since $c=2$ implies $(x-y)^{2}= \pm 2$, we obtain $c>2$. Dividing by $(x, y)$, we obtain an equation

$$
u^{2}-c u v+v^{2}= \pm c_{0},
$$

where $u, v \in \mathbf{Z},(u, v)=1, c_{0}>1$ and $c=c_{0} q^{2}$ for some $q \in \mathbf{N}$. If $D=c^{2}$ -4 , then $D$ is a discriminant, and

$$
\pm c_{0}=\mathscr{N}\left(\frac{2 u-c v+v \sqrt{D}}{2}\right)
$$

whence $c_{0} \in \Omega(D)$. If $4 \mid c_{0}$, then we obtain $u^{2}+v^{2} \equiv 0 \bmod 4$, contradicting $(u, v)=1$; therefore we have $4 \nmid c_{0}$ and thus $\left(c_{0}, f_{D}\right)=1$.

If $c_{0} \neq c$, then $c_{0} \leq c / 4<\frac{1}{2} \sqrt{D}$ and therefore $c_{0} \in \Omega^{*}(D)$. By [2], we have $\Omega^{*}(D)=\{1, c-2\}$ and therefore $c_{0}=1$, a contradiction.

If $c_{0}=c$ is odd, then Proposition 3, ii) implies $c=5$. If $c_{0}=c$ is even, then $c \equiv 2 \bmod 4$, since $4 \nmid c_{0}$, and therefore $u^{2}-c u v+v^{2} \equiv(u-v)^{2}$ $\equiv 2 \bmod 4$, a contradiction.

\section{REFERENCES}

[1] P. G. L. Dirichlet, Vorlesungen über Zahlentheorie, Braunschweig 1893, Chelsea Reprint 1968.

[2] F. Halter-Koch, Quadratische Ordnungen mit großer Klassenzahl, J. Number Theory, 34 (1990), 82-94.

[3] P. Kaplan, K. S. Williams, The distance between ideals in the orders of a real quadratic field, L'Enseig. Math., 36 (1990), 321-358.

[4] R. A. Mollin, On the insolubility of a class of Diophantine equations and the nontriviality of the class numbers of related real quadratic fields of Richaud-Degerttype, Nagoya Math. J., 105 (1987), 39-47.

[5] H. Yokoi, On the diophantine equation $x^{2}-p y^{2}= \pm 4 q$ and the class number of real subfields of a cyclotomic field, Nagoya Math. J., 91 (1983), 151-161.

Institut für Mathematik

Karl-Franzens-Universität

Halbärthgasse 1/I

A-8010 Graz, Österreich 